\title{
Challenges of providing treatment and care to men who have sex with men and with HIV/AIDS in Bangkok
}

\author{
Andrew Tam ${ }^{\mathrm{a}}$, Jennifer $\mathrm{Ho}^{\mathrm{b}}$, Annette H. Sohn ${ }^{\mathrm{b}}$ \\ ${ }^{a}$ UCSF Global Health Sciences, School of Medicine, University of California, San Francisco, CA, \\ USA, ${ }^{b}$ TREAT Asia/ amfAR-The Foundation for AIDS Research, Bangkok 10110, Thailand
}

\begin{abstract}
Background: Across the Asia-Pacific, men who have sex with men (MSM) constitute a growing proportion of those infected with and at risk for HIV/AIDS, but frequently lack access to treatment-related resources because of stigma and discrimination.

Objective: To identify challenges that a community-based organization (CBO) and its corresponding HIV clinic in Bangkok, Thailand, face in order to facilitate access to HIV-related care and treatment services by HIV-positive MSM.

Methods: Data were collected through focus group discussions, semi-structured interviews, and surveys of staff members and volunteers working at the support facilities from April through May 2011.

Results: A total of 21 staff and volunteers working at the support facilities participated. Participants reported various barriers to use of HIV-related services by MSM including fear of stigmatization because of their infection status, limited clinic/hospital hours, and misunderstanding of risks.

Conclusions: In response to these barriers, CBOs implemented solutions ranging from outreach activities to organization of a men's health clinic targeting MSM.
\end{abstract}

Keywords: Community, HIV/AIDS, MSM, stigma, Thailand

\section{HIV/AIDS epidemic in Thailand}

Despite the relatively low overall prevalence of HIV/AIDS when compared to the high prevalence in countries in Sub-Saharan Africa, the number of people who are infected with HIV in Asia continues to rise in the context of largely concentrated epidemics [1]. According to UNAIDS, approximately 280,000 people in South and Southeast Asia were newly infected with HIV in 2011, bringing the total number of people living with HIV/AIDS in the region to 4.0 million [2].

The Thai government implemented the " 100 Percent Condom Use in Commercial Sex Programme" in the 1990s to promote condom use in brothels [3]. The campaign was successful in curbing HIV infection among female sex workers and their clients, and HIV prevalence started to fall in the general population a few years later [4]. Epidemiological indicators revealed a decline in HIV infections among military recruits from 4\% in 1993 to

Correspondence to: Annette H. Sohn, TREAT Asia/ amfARThe Foundation for AIDS Research, 388 Sukhumvit Road, Suite 210, Bangkok 10110, Thailand. E-mail: annette.sohn@ treatasia.org
$0.5 \%$ in 2001, and among pregnant women from $2.3 \%$ in 1995 to $1.0 \%$ in 2004 [5]. As a result, the number of new infections decreased from 143,000 cases in 1991 to 19,000 cases in 2003 [4]. Despite the decline of HIV infections, Thailand continues to have one of the highest national prevalences of HIV/AIDS in Asia. It is estimated that the prevalence of HIV/AIDS was 1.2\% in 2011 and $1.1 \%$ in 2012 among adults aged 15-49 years compared with less than $1 \%$ in most other Asian countries [6, 7]. UNAIDS estimated 21,000 deaths in Thailand because of AIDS in 2012.

\section{Men who have sex with men living with HIV in Thailand}

Against the background of low and declining overall adult HIV prevalence in Asia, the population of men who have sex with men (MSM) continues to have substantially higher and increasing rates of HIV infection than the general public [5]. Thailand was one of the first countries in Asia to implement a national plan for HIV/AIDS, including a commitment to provide universal access to antiretroviral drugs [8]. However, MSM were not specifically identified in the national HIV/AIDS plan and HIV surveillance system until $2007[3,8]$. 
According to national MSM population estimations, approximately 120,000 MSM of a total of 550,000 MSM in Thailand are living in Bangkok (personal communication, N. Phanuphak). The two most well-known local clinics that provide HIV outreach and testing services targeted to the MSM community are the Silom Community Clinic (supported through the Thai-US Centers for Disease Control and Prevention Collaboration) and the Anonymous Clinic at the Thai Red Cross AIDS Research Centre. In 2011, out of 5,972 HIV tests among self-reported MSM conducted in Bangkok and reimbursed through the Thai National Health Security Organization, 96\% were at these two clinics (unpublished data). However, from a 2012 survey conducted among 5624 MSM in Bangkok by the Rainbow Sky Association of Thailand, 43\% stated they had their HIV tests done at private hospitals, outside of the public health and national insurance systems (personal communication, $\mathrm{N}$. Phanuphak).

Based on cross-sectional surveys, the HIV prevalence among MSM in Bangkok, Thailand has increased from 17\% in 2003 to $28 \%$ in 2005, and further to $31 \%$ in $2010[10,11]$. The Silom Community Clinic reported increased rates of HIV prevalence among MSM attending the clinic from 25\% in 2005 to $35 \%$ in 2010 [10]. A cohort study of MSM in Bangkok found an HIV incidence of 5.9 per 100 person-years between 2006 and 2011, an incidence among the highest reported since the initial outbreak in the Western world [12]. However, because many MSM may not report their sexual orientation to healthcare providers or HIV testing centers because of fear of stigma [8], prevalence of HIV/AIDS among MSM maybe under-reported [4]. HIV-positive MSM in Thailand experience stigma associated with being MSM and significant discrimination because of their HIV status [8], putting them at risk for poorer healthcare utilization and negative health outcomes [13].

\section{The challenges of CBOs supporting MSM with HIV/AIDS in Thailand}

The challenges of delivering treatment and care to MSM living with HIV/AIDS are under studied, in part because of difficulties in collecting data among HIV-positive MSM, particularly in resource-limited settings [14]. Public health programs seeking to initiate contact with MSM to help those who are living with HIV/AIDS struggle to identify effective, on-the- ground interventions to provide care to this population. Despite the high numbers of MSM within the overall HIV epidemic in Thailand, there are few clinics that specifically aim to deliver HIV services to MSM [15]. Community-based organizations (CBOs) act as a critical link between MSM in the community and local HIV care and treatment resources. They can help guide clients through healthcare systems, and advocate on their behalf. We conducted a survey of a CBO and one of its corresponding HIV clinics in Bangkok to study the factors that promote or hinder CBOs from initiating contact with HIV-positive MSM and connecting them to care.

\section{Setting}

\section{The Poz Home Center}

The Poz Home Center is located on the outskirts of Bangkok and opened its doors in 2005. It serves to connect people living with HIV (with a particular focus on HIV-positive MSM and male sex workers) to HIV care by providing a range of services such as offering those traveling from outside of Bangkok (and their families) a safe place to stay and accompanying them to their clinic visits. The Poz Home Center also provides other services, including peer counseling, drop-in counseling services, a telephone help line, and various education programs aimed to help clients to manage their disease better.

One of the most popular activities at The Poz Home Center has been a monthly group dinner. The group provides a supportive space where clients can share their experiences and knowledge with each other. They also organize various outreach activities, including participating in local holiday festivals to promote their organization.

\section{HIV-NAT/Thai Red Cross AIDS Research Centre}

The HIV Netherlands-Australia-Thailand Research Collaboration (HIV-NAT) was initially established in Bangkok in 1996 to coordinate and conduct clinical research on HIV and related infections. HIV-NAT, in collaboration with the Thai Red Cross AIDS Research Centre, also provides HIV testing, treatment, and care services to the general public at its "Anonymous Clinic." This is one of the clinics that The Poz Home Center refers their clients to for HIV care services.

This study focused on three components of the Anonymous Clinic: a mobile clinic, the Wednesday Friends' Club, and the Men's Health Clinic. 
- The mobile clinic travels to the community to educate and provide a wide range of HIV-related clinical and educational services. The mobile clinic team uses a large trailer to travel out to known MSM social venues in Bangkok to distribute condoms, lubricant, and educational materials, as well as conduct HIV testing.

- The Wednesday Friends' Club is a support group formed in 1990 that provides counseling services, vocational training, and support to people living with HIV. Club members also collaborate with local opinion leaders and organize lectures to inform people about the treatment and healthcare services that the clinic offers.

- $\quad$ The Men's Health Clinic was established in 2008 to provide HIV testing and counseling, screening and treatment of sexually transmitted infections, and general medical services for MSM. All clients registering at the Anonymous Clinic who report maleto-male sex on an intake questionnaire are referred to the Men's Health Clinic.

\section{Methods}

In our mixed-methods design, we conducted a focus group discussion (FGD) with staff and volunteers organizations; the project was considered an operational audit and service evaluation of staff and volunteer worker experiences and opinions. The project was conducted according to the current principles of the Declaration of Helsinki and was consistent with guidelines from the Committee for Publication Research Ethics (COPE) guidance: Research, Audit and Service Evaluations.

\section{Focus group discussion}

We conducted one focus group discussion (FGD) with the staff and volunteer workers of The Poz Home Center to assess factors impacting client use of services at The Poz Home Center and at local clinics or hospitals. Participants provided verbal consent to participate in and allow for audio recording of the FGD. This nonwritten consent was witnessed and formally documented (archived on tape). The majority of the discussion was conducted in Thai with translation to English, and was developed around seven questions, which were written on posters in the room where the FGD took place.

\section{FGD questions}

- What are the reasons why clients would or would not come to Poz Home for support and resources?

- What do you think are the factors that prevent clients from returning to Poz Home?

- What are the reasons why clients would or would not go the clinic/hospital for HIV care and health services?

- What do you think are the factors that prevent HIV-positive MSM clients from returning to the clinic/hospital for follow-up appointments and HIV care?

- What interventions has Poz Home attempted in bringing clients in for support and resources?

- What interventions had Poz Home attempted to ensure that the clients are returning to the clinic/hospital for HIV care and sexual health services?

- What were or have been the difficulties in implementing these interventions?

who work at The Poz Home Center. We also conducted semi-structured interviews with an outreach worker from the mobile clinic, a peer group member from the Wednesday Friends' Club, and a counselor from the Men's Health Clinic of the Anonymous Clinic of HIV-NAT/Thai Red Cross. Surveys were then developed based on the common themes identified through the FGD and semistructured interviews, and were distributed to staff and volunteer workers of both organizations. Ethics committee review was not sought by the participating
During the FGD, participants were asked to respond to each question by writing down their responses and posting them on each of the corresponding posters. With the assistance of the translator, we then combined the responses based on their general content area. The group then discussed each of the content areas and was asked to further elaborate on the major themes. Names and other identifying information were removed from audio transcripts that were anonymized to ensure confidentiality. 


\section{Semi-structured interviews}

Key informants were asked to participate in a semi-structured interview that addressed factors impacting the use of services by MSM clients at the clinic. These key informants represented the departments that focused on providing support services to the MSM community (e.g., outreach, peer support, counseling) and were recommended by the clinic's head physician. Participants provided verbal consent to be interviewed and allow for audio recording. This nonwritten consent was witnessed and formally documented (archived on tape). Interviews were conducted in Thai in private offices with translation to English. The interviews were audio recorded and later transcribed. Names and other identifying information were removed from the transcripts that were anonymized to ensure confidentiality before analysis.

\section{Cross-sectional quantitative survey}

A survey based on common themes from the FGD and the semi-structured interviews was developed, translated into Thai, and sent to the program directors of The Poz Home Center and the Anonymous Clinic to distribute to staff members and program volunteers working with MSM. Program directors were instructed to allow staff members and volunteer workers to complete the surveys independently without being supervised. The Poz Home Center survey had three sections that asked about factors that impacted (1) the operation of Poz Home, (2) client use of the Poz Home services, and (3) client use of HIV treatment and care at local clinics or hospitals. The Anonymous Clinic survey had two sections that asked about factors that that impacted (1) the operation of the clinic, and (2) client use of HIV and health services. Both surveys used a Likert scale in asking participants to review a statement and to indicate their evaluation on a scale of 1 (strongly disagree) to 6 (strongly agree) as shown in Table 1. An even-numbered Likert scale was used to remove the neutral option in order to better characterize respondents' opinions and attitudes.

Table 1. Survey responses of staff and volunteers from The Poz Home Center and the Anonymous Clinic of HIV-NAT/ Thai Red Cross AIDS Research Centre $(n=21)$

\begin{tabular}{|c|c|c|c|}
\hline Survey Item & $\begin{array}{l}\text { Poz Home } \\
\text { (mean, SD) }\end{array}$ & $\begin{array}{l}\text { Anonymous Clinic } \\
\text { (mean, SD) }\end{array}$ & $\boldsymbol{P}$ \\
\hline $\begin{array}{l}\text { Funding supporting the organization and its activities is adequate } \\
\text { to meet program and client needs. }\end{array}$ & $3.86(1.07)$ & $3.33(2.12)$ & 0.67 \\
\hline The organization is understaffed to meet program and client needs. & $3.29(1.80)$ & $4.89(1.27)$ & 0.05 \\
\hline $\begin{array}{l}\text { The organization has been successful in reaching out to the MSM } \\
\text { and HIV communities in Bangkok. }\end{array}$ & $4.86(1.46)$ & $4.00(1.73)$ & 0.28 \\
\hline $\begin{array}{l}\text { The organization has strong support from the general community } \\
\text { (outside of the MSM and HIV communities). }\end{array}$ & $4.86(1.46)$ & $4.78(1.30)$ & 0.78 \\
\hline $\begin{array}{l}\text { Clients come to Poz Home because Poz Home provides the support } \\
\text { and resources that clients are looking for. }\end{array}$ & $4.63(1.19)$ & N/A & N/A \\
\hline $\begin{array}{l}\text { It is convenient for clients to access support and resources from } \\
\text { Poz Home. }\end{array}$ & $4.63(1.19)$ & N/A & N/A \\
\hline $\begin{array}{l}\text { Clients are afraid to be seen coming to an organization that } \\
\text { provides support and resources to MSM. }\end{array}$ & $3.00(1.20)$ & N/A & N/A \\
\hline $\begin{array}{l}\text { Clients are afraid to be seen coming to an organization that } \\
\text { provides support and resources to people living with HIV. }\end{array}$ & $3.50(1.77)$ & N/A & N/A \\
\hline $\begin{array}{l}\text { Limited operating hours at Poz Home make it difficult for clients } \\
\text { to access support and resources. }\end{array}$ & $4.00(1.51)$ & N/A & N/A \\
\hline $\begin{array}{l}\text { Clients feel welcome by the staff members and volunteers at Poz } \\
\text { Home. }\end{array}$ & $5.13(1.36)$ & N/A & N/A \\
\hline $\begin{array}{l}\text { Clients are made to feel welcome by staff members at their local } \\
\text { clinic/hospital. }\end{array}$ & $4.43(1.40)$ & $4.92(1.04)$ & 0.33 \\
\hline $\begin{array}{l}\text { Clients feel that providers at their local clinic/hospital treat them } \\
\text { with respect. }\end{array}$ & $4.43(1.40)$ & $5.23(0.60)$ & 0.25 \\
\hline Clients do not see the need to obtain HIV treatment and care. & $3.14(1.68)$ & $2.00(1.47)$ & 0.09 \\
\hline $\begin{array}{l}\text { Clients are afraid to be seen at local clinics/hospitals seeking HIV } \\
\text { treatment and care. }\end{array}$ & $4.71(0.95)$ & $3.54(1.98)$ & 0.25 \\
\hline $\begin{array}{l}\text { Limited clinic/hospital hours make it difficult for clients to access } \\
\text { care and treatment. }\end{array}$ & $4.71(1.25)$ & $3.08(2.10)$ & 0.10 \\
\hline $\begin{array}{l}\text { It is convenient for clients to travel to their local clinic/hospital } \\
\text { for HIV treatment and care. }\end{array}$ & $4.29(1.25)$ & $4.31(1.60)$ & 0.79 \\
\hline
\end{tabular}


The survey was anonymous and voluntary, without remuneration or negative consequences for agreeing or declining to participate. Participants were informed that completed surveys would be analyzed and the data used for the purpose of this study.

\section{Results \\ Sample description}

Poz Home

The FGD held at Poz Home included three staff members and seven part-time volunteer workers. All participants were Thai and self-identified as MSM, except for one participant who self-identified as transgender. Surveys were distributed to seven Poz Home staff and three volunteers; eight were completed. The eight participants ranged in work experience at Poz Home from less than six months to four years, with a mean of one year. The surveys showed that seven of eight participants had direct interaction with clients as part of their responsibilities at Poz Home. Of the seven, the average mean time they spent with clients was 1-3 hours per day. Their primary responsibilities at Poz Home ranged from managing activities of the organization to providing counseling by phone.

The Anonymous Clinic of HIV-NAT/Thai Red Cross

The work experience of the three interview participants at the clinic ranged from 5-14 years, with a mean of 8.3 years. All participants were Thai and self-identified as MSM. Surveys were distributed to 18 members of the mobile clinic staff, the Wednesday Friends' Club, and the Men's Health Clinic; 13 surveys were completed. Self-reported work experience ranged from six months to 14 years, with a mean of four years. Surveys showed that 10 participants had direct interaction with clients; the mean time spent with clients was five hours per day.

\section{Qualitative findings}

\section{Poz Home}

Factors impacting client utilization of $\mathrm{Poz}$ Home services

Participants of the FGD were asked to identify reasons why new or current clients would or would not use Poz Home support and resources. There was a consensus from the group that clients came mainly for the following reasons: (1) to understand treatment and care, (2) to gain new or updated information, (3) because the staff would take them to meet doctors and facilitate follow-up. There were also several participants who described meeting friends as their reason for coming to Poz Home.

As for reasons why clients would not seek out Poz Home support, participants identified the distance clients had to travel to get to the facility as a major factor. Other barriers included (1) fear that they would be harassed or someone would know that they had HIV, (2) they already had received help, and (3) they were not satisfied with some aspects of Poz Home's available services.

Factors impacting client utilization of HIV care and health services from local clinics/hospitals

Poz Home participants described a major factor in preventing their clients from seeking services from local clinics/hospitals was fear of unwanted disclosure of their HIV disease status in general:

"First, (they are) afraid that someone else will find out (about their HIV status) and second, afraid to learn that their health might have worsen. Also because not everyone understands HIV/AIDS, they are afraid that somebody will show undesirable feeling toward (them)."

\section{Another participant noted:}

"How to maintain their health? How to deal with family after that...how much it cost for treatment and where to access healthcare services for people living with HIV? Sometimes they actually know they already transmitted HIV but they just ignore and let their life go on."

When asked if disclosure of sexual practices had a role in client utilization of HIV-related healthcare and support services, one participant stated that it was not a major barrier.

\section{The Anonymous Clinic of HIV-NAT/Thai Red Cross}

Factors impacting use of HIV care and health services

Staff capacity was identified during the key informant interviews as a primary barrier to service delivery for MSM. The client load at the Men's Health Clinic was considered by those interviewed to be high for the number of paid full-time staff, often leading to outreach staff having to recruit volunteer workers to 
assist with outreach activities or inadequate coverage. It was also noted that it had been difficult retaining experienced staff members because of the lack of financial incentives and long work hours.

Overall, the general atmosphere of most healthcare facilities in Bangkok was viewed as a barrier to providing healthcare services to MSM. One interviewee described the atmosphere of other clinics as "very formal" and that "you feel like you did something bad and that's why you had to come here."

Both the outreach worker and peer group representative identified misunderstanding of risks as a reason why clients would not go the clinic to get tested for HIV. One example the outreach worker provided was a misconception that his teenage clients often had:

"They thought [that the] HIV virus sleeps during the day... if they have unprotected sex during the day they are safe because HIV is not working."

The outreach worker and the counselor both noted that some MSM did not admit that they were MSM. The work hours of some MSM clients were also identified by the peer group representative as a barrier for these individuals to obtain services from the clinic:

"MSM who are sex workers, their work hours are so different. Most work at night and rest during the day. When they feel like going to clinic, it might not be during the hours of clinic."

Fear was consistently viewed as the most common reason why MSM would not seek HIV-related services. Drawing from their experiences of working with the MSM community, they identified three kinds of fear that would deter clients from going the clinic:

(1) Fear of knowing that they were HIV-positive.

"Some people think that if they come in to test and found out that they are positive, then they don't know what to do. So like, not knowing at all might be better."-Peer group representative

"The old messages of HIV associated with people getting very sick and weak; with all the 'things' happening to your body. People think HIV is something scary."-Outreach worker
(2) Fear of meeting people that they might know at the clinic.

"They don't want anyone to know that they come to the clinic...they fear that if they come into the group, they meet people that will know their status."-Peer group representative

(3) Fear of coming to a clinic such as the Anonymous Clinic because it was often associated with HIV.

"The name of Anonymous [Clinic] is a specific name, most people know that the Anonymous Clinic treats specifically for HIV."-Counselor

\section{Survey findings}

Survey results showed that staff members from both organizations moderately to strongly agreed (mean score ranging from 4.00 to 4.86 ) that their organizations had been successful in reaching out or initiating contact with MSM and people living with HIV to offer help and had strong support from the general community (Table 1). The most strongly rated operational issue for HIV-NAT/Thai Red Cross staff was they felt that the organization was understaffed in meeting the program and client need (mean score $=4.89$ ).

Poz Home respondents strongly rated their success in reaching out to MSM and people living with HIV in Bangkok and building support from the general community (mean score $=4.86$ ). They affirmed their belief that they made clients feel welcome (mean score $=5.13$ ), which was the highest scored item on their surveys.

With regards to the clinical services the Anonymous Clinic or other local clinics or hospitals provided, the highest agreements among HIV-NAT/ Thai Red Cross staff was for the statement that "clients feel that providers at their local clinicl hospital treat them with respect" (mean score $=5.23$ ) and the lowest agreement was for the statement "clients do not see the need to obtain HIV treatment and care" with a mean score of 2.00. Poz Home respondents rated the statements regarding not seeing the need to obtain HIV treatment and care services the lowest as well (mean score $=3.14$ ). Statements with the highest agreements among Poz Home respondents were "clients are afraid to be seen at local clinics/hospitals seeking HIV treatment and care" and "limited clinic/hospital hours make it difficult for clients to access care and treatment"; both with a mean score of 4.71 . 


\section{Discussion}

The persistent rise in HIV infections among MSM in Asia has led to a growing recognition of the need to facilitate better access to and uptake of healthcare services for MSM in the Asia-Pacific region (12). The primary objective of this descriptive study was to use a mixed-methods approach to understand barriers to service use within a CBO and an MSM-focused clinic in Bangkok. This mixed-methods approach allowed us to obtain valuable qualitative data from those who work closely with the MSM population and gain new perspectives to guide future endeavors for HIV prevention that would not have been revealed from quantitative patient data alone.

One of the most consistent qualitative findings was that reduced uptake of healthcare-related resources by MSM in the community was linked to fear of knowing their own HIV status and of public disclosure of their infection status. This fear of finding out that they were HIV-positive was intertwined with the fear of not being able to access treatment, and of how the disease would impact their relationships with their families and partners. One of the key informant interviewees also identified the negative attitudes of providers as a factor in deterring clients from returning to HIV clinics. This finding is consistent with another study conducted with MSM and transgender women in Thailand that reported high rates of perceived hostility from healthcare providers and being given less attention than other patients [13]. These results suggest that future interventions should include efforts to reduce discrimination in the healthcare system.

However, our data did not directly point to fear of stigma associated with their sexual practices as a major barrier to uptake of healthcare and support services. Both The Poz Home Center and the Anonymous Clinic had services provided specifically to MSM clients. Although those clients who were fearful of people finding out that they were MSM might choose not to disclose their true sexual practices or avoid organizations that exclusively provided care for MSM, the benefits of MSM-sensitive services appeared to facilitate use of HIV-related healthcare and support services.

Poz Home staff and volunteer workers identified limited clinic/hospital hours as the one of the most important barriers impacting client use of services. Despite this not being an issue raised during the FGD, the inability to extend clinic hours to evenings and weekends has been an ongoing struggle for conventional HIV clinics in public healthcare settings.
Related to this issue are the limitations in staff coverage, as reported by the clinic survey participants and in the interviews of an outreach worker and a counselor. Nevertheless, most of the staff and volunteers "generally agreed" (scores over 4) with most of the positive statements concerning the quality of their services.

There are several areas where biases might have been introduced during the study. Because the organizations were relatively small, similar individuals were identified in a nonrandom way to participate in the interviews, FGD, and the surveys. Therefore, it is possible that our survey data are not independent of the qualitative findings. Moreover, in our FGD, many volunteer workers were past clients of the organizations and therefore reflected on their own experiences, which may not fully reflect the experiences of current clients, and could be biased in favor of the organization. However, it is also notable that past clients were confident enough in the quality of the organization that they chose to volunteer there as staff. More broadly, data were collected from a small number of participants, which limits the generalizability of the results to the greater HIV community in Bangkok and the statistical power to find major differences in how the two groups rated the items on the survey. Topics such as the cost of HIV treatment and distance to travel to obtain services were not included in the survey. In addition, this study focused on the attitudes of nonmedical staff and volunteer workers, while clients and healthcare providers such as physicians were not included. Despite these limitations, the study brings to light perspectives of CBOs and their challenges of reaching out to MSM living with HIV in Thailand.

CBOs and HIV clinics in Bangkok are confronted daily with the challenge of addressing barriers to use of HIV-related services by MSM. The Poz Home Center and the Anonymous Clinic are examples of organizations that have attempted to address these barriers through outreach activities aimed at educating the public about HIV and the importance of getting tested, establishing an MSM-focused clinic, providing services during expanded hours, and offering a variety of counseling services. Collectively, these interventions include individual-, group-, and community-level interventions to facilitate access to prevention, care, and treatment of HIV. Better understanding of the complexities of these issues is necessary to facilitate more effective implementation of HIV prevention interventions for MSM. 


\section{Acknowledgments}

The authors thank Dr. Praphan Phanuphak, Dr. Nittaya Phanuphak, Mr. Somchai Phromsombut, and the staff and volunteer workers at The Poz Home Center Foundation and the Anonymous Clinic, HIVNAT/Thai Red Cross AIDS Research Centre, for their participation in this project, and Dr. Wayne Steward from the University of California, San Francisco, for his helpful comments on earlier drafts of the manuscript. Additional thanks go to Noweed Charles, Apple Pethpoowong, May Kaewchang, Tom Serkpookiaw, and Nada Chaiyajit for their assistance in the field.

None of the authors has any conflict of interest to report.

\section{References}

1. Bridge J, Lazarus JV, Atun R. HIV epidemics and prevention responses in Asia and Eastern Europe: lessons to be learned? AIDS. 2010; 24 (Suppl 3): S86-94.

2. UNAIDS. 2012 UNAIDS Report on the Global AIDS Epidemic; 2012.

3. Celentano DD. Undocumented epidemics of HIV continue to persist in the twenty-first century. AIDS. 2005; 19:527-8.

4. van Griensven F, Thanprasertsuk S, Jommaroeng R. Evidence of a previously undocumented epidemic of HIV infection among men who have sex with men in Bangkok, Thailand. AIDS. 2005; 19:521-6.

5. van Griensven F, de Lind van Wijngaarden JW. A review of the epidemiology of HIV infection and prevention responses among MSM in Asia. AIDS. 2010; 24 (Suppl 3):S30-40.

6. UNAIDS. UNAIDS: HIV and AIDS estimates 2011Thailand. [online] 2012 [cited 2013 Jan 19]. Available from http://www.unaids.org/en/regionscountries/ countries/thailand/
7. UNAIDS. UNAIDS: HIV and AIDS estimates 2012Thailand. [online] 2013 [cited 2013 Jan 19]. Available from http://www.unaids.org/en/regionscountries/ countries/thailand/

8. Beyrer C, Baral SD, Walker D, Wirtz AL, Johns B, Sifakis F. The expanding epidemics of HIV type 1 among men who have sex with men in low- and middle-income countries: diversity and consistency. Epidemiol Rev. 2010; 32:137-51.

9. IRIN Asia. Thailand's MSM face alarming HIV ratesThailand-HIV/AIDS (PlusNews). [online] 2013

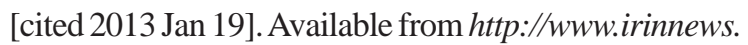
org/report/98439/thailand-s-msm-face-alarminghiv-rates

10. USAID. Men who have sex with men and transgender population: multi-city initiative. [online] 2010 [cited 2013 Jan 19]. Available from http://www.snap-undp. org/elibrary/Publication. aspx ?id $=538$

11. Centers for Disease Control and Prevention (CDC). HIV and syphilis infection among men who have sex with men-Bangkok, Thailand, 2005-2011. MMWR Morb Mortal Wkly Rep. 2013; 62:518-20.

12. van Griensven F, Thienkrua W, McNicholl J, Wimonsate W, Chaikummao S, Chonwattana W, et al. Evidence of an explosive epidemic of HIV infection in a cohort of men who have sex with men in Bangkok, Thailand. AIDS. 2013; 27:825-32.

13. Dowshen N, Binns HJ, Garofalo R. Experiences of HIV-related stigma among young men who have sex with men. AIDS Patient Care STDS. 2009; 23:371-6.

14. Vanlandingham M, Im-Em W, Yokota F. Access to treatment and care associated with HIV infection among members of AIDS support groups in Thailand. AIDS Care. 2006; 18:637-46.

15. Sirivongrangson $P$, Lolekha $R$, Charoenwatanachokchai A. HIV risk behavior among HIV-infected men who have sex with men in Bangkok, Thailand. AIDS Behav. 2012; 16:618-25. 